\title{
Dependency-Based Bilingual Language Models for Reordering in Statistical Machine Translation
}

\author{
Ekaterina Garmash and Christof Monz \\ Informatics Institute, University of Amsterdam \\ Science Park 904, 1098 XH Amsterdam, The Netherlands \\ $\{$ e.garmash, c.monz\}@uva.nl
}

\begin{abstract}
This paper presents a novel approach to improve reordering in phrase-based machine translation by using richer, syntactic representations of units of bilingual language models (BiLMs). Our method to include syntactic information is simple in implementation and requires minimal changes in the decoding algorithm. The approach is evaluated in a series of ArabicEnglish and Chinese-English translation experiments. The best models demonstrate significant improvements in BLEU and TER over the phrase-based baseline, as well as over the lexicalized BiLM by Niehues et al. (2011). Further improvements of up to 0.45 BLEU for ArabicEnglish and up to 0.59 BLEU for ChineseEnglish are obtained by combining our dependency BiLM with a lexicalized BiLM. An improvement of 0.98 BLEU is obtained for Chinese-English in the setting of an increased distortion limit.
\end{abstract}

\section{Introduction}

In statistical machine translation (SMT) reordering (also called distortion) refers to the order in which source words are translated to generate the translation in the target language. Word orders can differ significantly across languages. For instance, Arabic declarative sentences can be verbinitial, while the corresponding English translation should realize the verb after the subject, hence requiring a reordering. Determining the correct reordering during decoding is a major challenge for SMT. This problem has received a lot of attention in the literature (see, e.g., Tillmann (2004), Zens and Ney (2003), Al-Onaizan and Papineni (2006)), as choosing the correct reordering improves readability of the translation and can have a substantial impact on translation quality (Birch, 2011). In this paper, we only consider those approaches that include a reordering feature function into the loglinear interpolation used during decoding.

The simplest reordering model is linear distortion (Koehn et al., 2003) which scores the distance between phrases translated at steps $t$ and $t+1$ of the derivation. This model ignores any contextual information, as the distance between translated phrases is its only parameter. Lexical distortion modeling (Tillmann, 2004) conditions reordering probabilities on the phrase pairs translated at the current and previous steps. Unlike linear distortion, it characterizes reordering not in terms of distance but type: monotone, swap, or discontinuous.

In this paper, we base our approach to reordering on bilingual language models (Marino et al., 2006; Niehues et al., 2011). Instead of directly characterizing reordering, they model sequences of elementary translation events as a Markov process. ${ }^{1}$ Originally, Marino et al. (2006) used this kind of model as the translation model, while more recently it has been used as an additional model in PBSMT systems (Niehues et al., 2011). We adopt and generalize the approach of Niehues et al. (2011) to investigate several variations of bilingual language models. Our method consists of labeling elementary translation events (tokens of bilingual LMs) with their different contextual properties.

What kind of contextual information should be incorporated in a reordering model? Lexical information has been used by Tillmann (2004) but is known to suffer from data sparsity (Galley and Manning, 2008). Also previous contributions to bilingual language modeling (Marino et al., 2006; Niehues et al., 2011) have mostly used lexical information, although Crego and Yvon (2010a) and Crego and Yvon (2010b) label bilingual to-

\footnotetext{
${ }^{1}$ Note that the standard PBSMT translation model assumes that events of translating separate phrases in a sentence are independent.
} 
kens with a rich set of POS tags. But in general, reordering is considered to be a syntactic phenomenon and thus the relevant features are syntactic (Fox, 2002; Cherry, 2008). Syntactic information is incorporated in tree-based approaches in SMT, allowing one to provide a more detailed definition of translation events and to redefine decoding as parsing of a source string (Liu et al., 2006; Huang et al., 2006; Marton and Resnik, 2008), of a target string (Shen et al., 2008), or both (Chiang, 2007; Chiang, 2010). Reordering is a result of a given derivation, and CYK-based decoding used in tree-based approaches is more syntax-aware than the simple PBSMT decoding algorithm. Although tree-based approaches potentially offer a more accurate model of translation, they are also a lot more complex and requiring more intricate optimization and estimation techniques (Huang and Mi, 2010).

Our idea is to keep the simplicity of PBSMT but move towards the expressiveness typical of treebased models. We incrementally build up the syntactic representation of a translation during decoding by adding precomputed fragments from the source parse tree. The idea to combine the merits of the two SMT paradigms has been proposed before, where Huang and Mi (2010) introduce incremental decoding for a tree-based model. On a very general level, our approach is similar to theirs in that it keeps track of a sequence of source syntactic subtrees that are being translated at consecutive decoding steps. An important difference is that they keep track of whether the visited subtrees have been fully translated, while in our approach, once a syntactic structural unit has been added to the history, it is not updated anymore.

In this paper, we focus on source syntactic information. During decoding we have full access to the source sentence, which allows us to obtain a better syntactic analysis (than for a partial sentence) and to precompute the units that the model operates with. We investigate the following research questions: How well can we capture reordering regularities of a language pair by incorporating source syntactic parameters into the units of a bilingual language model? What kind of source syntactic parameters are necessary and sufficient?

Our contributions can be summarized as follows: We argue that the contextual information used in the original bilingual models (Niehues et al., 2011) is insufficient and introduce a simple model that exploits source-side syntax to improve reordering (Sections 2 and 3). We perform a thorough comparison between different variants of our general model and compare them to the original approach. We carry out translation experiments on multiple test sets, two language pairs (ArabicEnglish and Chinese-English), and with respect to two metrics (BLEU and TER). Finally, we present a preliminary analysis of the reorderings resulting from the proposed models (Section 4).

\section{Motivation}

In this section, we elaborate on our research questions and provide background for our approach. We also discuss existing bilingual n-gram models and argue that they are often not expressive enough to differentiate between alternative reorderings. We should first note that the most commonly used n-gram model to distinguish between reorderings is a target language model, which does not take translation correspondence into account and just models target-side fluency. Al-Onaizan and Papineni (2006) show that target language models by themselves are not sufficient to correctly characterize reordering. In what follows we only discuss bilingual models.

The word-aligned sentence pair in Figure $1 . \mathrm{a}^{2}$ demonstrates a common Arabic-English reordering. As stated in the introduction, bilingual language models capture reordering regularities as a sequence of elementary translation events ${ }^{3}$. In the given example, one could decompose the sequential process of translation as follows: First translate the first word Alwzyr as the minister, then ArjE as attributed, then ArtfAE as the increase and so on. The sequence of elementary translation events is modeled as an n-gram model (Equation 1, where $t_{i}$ is a translation event). There are numerous ways in which $t_{i}$ can be defined. Below we first discuss how they have been defined within previous approaches, and then introduce our definition.

$$
p\left(t_{1}, \ldots, t_{m}\right)=\prod_{i=1}^{m} p\left(t_{i} \mid t_{i-n+1} \ldots t_{i-1}\right)
$$

\subsection{Lexicalized bilingual LMs}

By including both source and target information into the representation of translation events we ob-

\footnotetext{
${ }^{2}$ We used Buckwalter transliteration for Arabic words.

${ }^{3}$ By an elementary translation event we mean a translation of some substructure of a sentence.
} 


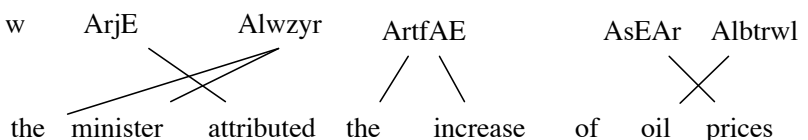

(a) The original word alignment.

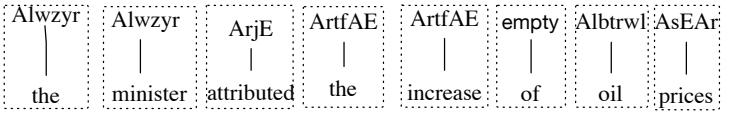

(b) BiLM tokens extracted from sentence (a).

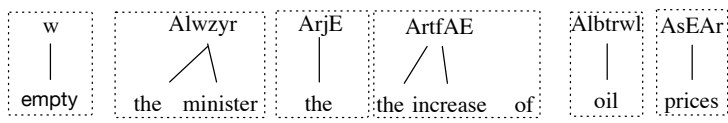

(c) MTU tokens extracted from sentence (a).

Figure 1: Arabic-English parallel sentence, automatically word-aligned. The bilingual token sequences are produced according to two alternative definitions (BiLM and MTU).

tain a bilingual LM. The richer representation allows for a finer distinction between reorderings. For example, Arabic has a morphological marker of definiteness on both nouns and adjectives. If we first translate a definite adjective and then an indefinite noun, it will probably not be a likely sequence according to the translation model. This kind of intuition underlies the model of Niehues et al. (2011), a bilingual LM (BiLM), which defines elementary translation events $t_{1}, \ldots, t_{n}$ as follows:

$$
t_{i}=\left\langle e_{i},\left\{f \mid f \in A\left(e_{i}\right)\right\}\right\rangle,
$$

where $e_{i}$ is the $i$-th target word and $A: E \rightarrow$ $\mathcal{P}(F)$ is an alignment function, $E$ and $F$ referring to target and source sentences, and $\mathcal{P}(\cdot)$ is the powerset function. In other words, the $i$-th translation event consists of the $i$-th target word and all source words aligned to it. Niehues et al. (2011) refer to the defined translation events $t_{i}$ as bilingual tokens and we adopt this terminology.

There are alternative definitions of bilingual language models. Our choice of the above definition is supported by the fact that it produces an unambiguous segmentation of a parallel sentence into tokens. Ambiguous segmentation is undesirable because it increases the token vocabulary, and thus the model sparsity. Another disadvantage comes from the fact that we want to compare permutations of the same set of elements. For example, the two different segmentations of $b a$ into $[b a]$ and $[b][a]$ still represent the same permutation of the sequence $a b$. In Figure 1 one can produce a segmentation of (AsEAr Albtrwl, oil prices) into (Albtrwl, oil) and (AsEAr, prices) or leave it as is. If we allow for both segmentations, the learnt probability parameters may be different for the sum of (Albtrwl, oil) and (AsEAr, prices) and for the unsegmented phrase.
Durrani et al. (2011) introduce an alternative method for unambiguous bilingual segmentation where tokens are defined as minimal phrases, called minimal translation units (MTUs). Figure 1 compares the BiLM and MTU tokenization for a specific example. Since Niehues et al. (2011) have shown their model to work successfully as an additional feature in combination with commonly used standard phrase-based features, we use their approach as the main point of reference and base our approach on their segmentation method. In the rest of the text we refer to Niehues et al. (2011) as the original BiLM. ${ }^{4}$ At the same time, we do not see any specific obstacles for combining our work with MTUs.

\subsection{Suitability of lexicalized BiLM to model reordering}

As mentioned in the introduction, lexical information is not very well-suited to capture reordering regularities. Consider Figure 2.a. The extracted sequence of bilingual tokens is produced by aligning source words with respect to target words (so that they are in the same order), as demonstrated by the shaded part of the picture. If we substituted the Arabic translation of Egyptian for the Arabic translation of Israeli, the reordering should remain the same. What matters for reordering is the syntactic role or context of a word. By using unnecessarily fine-grained categories we risk running into sparsity issues.

Niehues et al. (2011) also described an alternative variant of the original BiLM, where words are substituted by their POS tags (Figure 2.a, shaded part). Also, however, POS information by itself may be insufficiently expressive to separate cor-

\footnotetext{
${ }^{4}$ Although, strictly speaking, it is not the original approach (see the references in Section 1).
} 


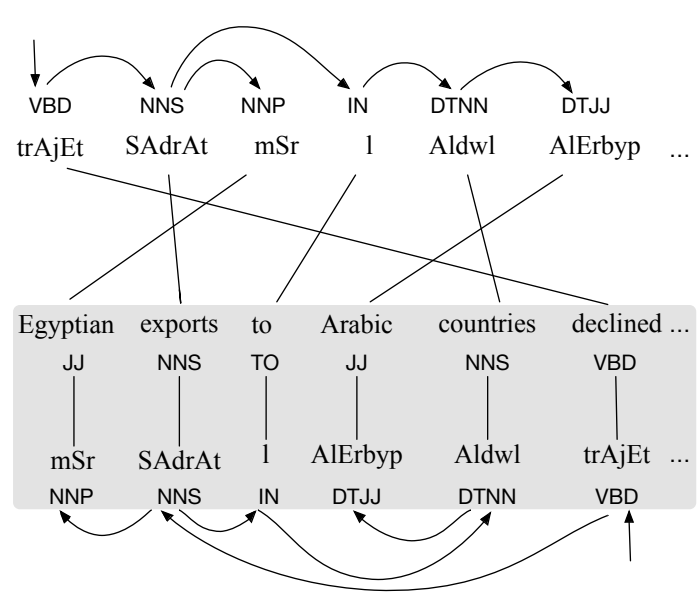

(a)

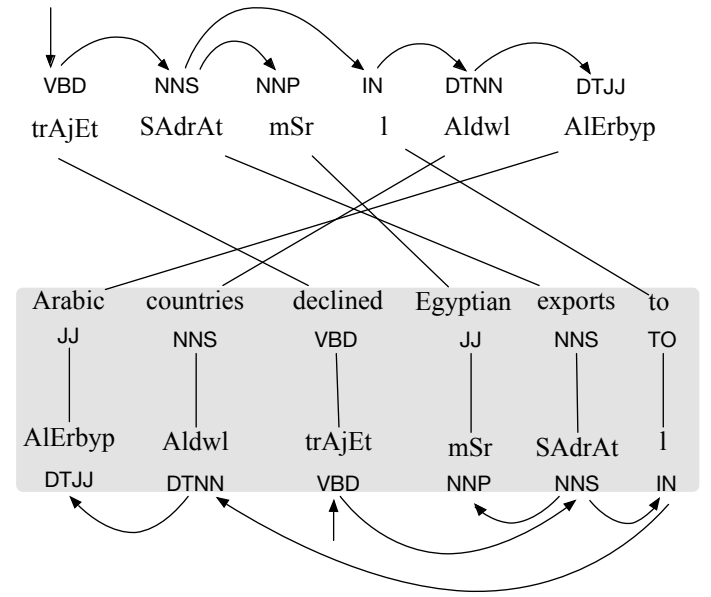

(b)

Figure 2: Arabic-English parallel sentence, automatically parsed and word-aligned, with corresponding sequences of bilingual tokens (in the shaded part). Comparison between translations produced via correct (a) and incorrect (b) reorderings.

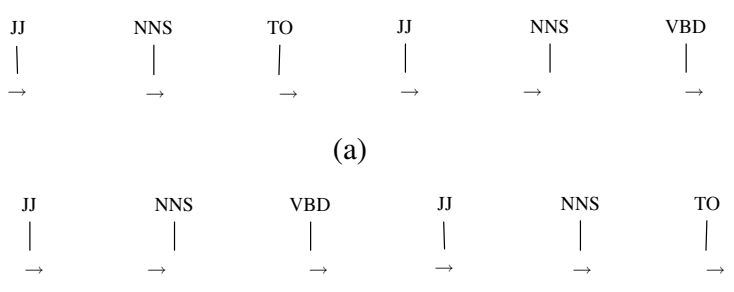

(b)

Figure 3: Sequences of bilingual tokens with source words substituted with their and their parents' POS tags: correct (a) and incorrect (b) reorderings.

rect and incorrect reorderings, see Figure 2.b. Although the corresponding sequence of POS-tagsubstituted bilingual tokens is different from the correct sequence (Figure 2.b, shaded part), it still is a likely sequence. Indeed, the log-probabilities of the two sequences with respect to a 4-gram BiLM model ${ }^{5}$ result in a higher probability of -10.25 for the incorrect reordering than for the correct one (-10.39).

Since fully lexicalized bilingual tokens suffer from data sparsity and POS-based bilingual tokens are insufficiently expressive, the question is which level of syntactic information strikes the right balance between expressiveness and generality.

\footnotetext{
${ }^{5}$ Section 4 contains details about data and software setup.
}

\subsection{BiLM with dependency information}

Dependency grammar is commonly used in NLP to formalize role-based relations between words. The intuitive notion of syntactic modification is captured by the primitive binary relation of dependence. Dependency relations do not change with the linear order of words (Figure 2) and therefore can provide a characterization of a word's syntactic class that invariant under reordering.

If we incorporate dependency relations into the representation of bilingual tokens, the incorrect reordering in Figure 2.b will produce a highly unlikely sequence. For example, we can substitute each source word with its POS tag and its parent's POS tag (Figure 3). Again, we computed 4-gram log-probabilities for the corresponding sequences: the correct reordering results in a substantially higher probability of -10.58 than the incorrect one $(-13.48)$. We may consider situations where more fine-grained distinctions are required. In the next section, we explore different representations based on source dependency trees.

\section{Dependency-based BiLM}

In this section, we introduce our model which combines the BiLM from Niehues et al. (2011) with source dependency information. We further give details on how the proposed models are trained and integrated into a phrase-based decoder. 


\subsection{The general framework}

In the previous section we outlined our framework as composed of two steps: First, a parallel sentence is tokenized according to the BiLM model (Niehues et al., 2011). Next, words in the bilingual tokens are substituted with their contextual properties. It is thus convenient to use the following generalized definition for a token sequence $t_{1} \ldots t_{n}$ in our framework:

$$
t_{i}=\left\langle\operatorname{Cont} E\left(e_{i}\right),\left\{\operatorname{Cont} F(f) \mid f \in A\left(e_{i}\right)\right\}\right\rangle,
$$

where $e_{i}$ is the $i$-th target word, $A: E \rightarrow \mathcal{P}(F)$ is an alignment function, $F$ and $E$ are source and target sentences, and ContE and ContF are target and source contextual functions, respectively. A contextual function returns a word's contextual property, based on its sentential context (source or target). See Figure 4 for an example of a sequence of BiLM tokens with a ContF defined as returning the POS tag of the source word combined with the POS tags of its parent, grandparent and siblings, and ContE defined as an identity function (see Section 3.2 for a detailed explanation of the functions and notation).

In this work we focus on source contextual functions $(C o n t F)$. We also exploit some very simple target contextual functions, but do not go into an in-depth exploration.

\subsection{Dependency-based contextual functions}

In NLP approaches exploiting dependency structure, two kinds of relations are of special importance: the parent-child relation and the sibling relation. Shen et al. (2008) work with two wellformed dependency structures, both of which are defined in such a way that there is one common parent and a set of siblings. Li et al. (2012) characterize rules in hierarchical SMT by labeling them with the POS tags of the parents of the words inside the rule. Lerner and Petrov (2013) model reordering as a sequence of classification steps based on a dependency parse of a sentence. Their model first decides how a word is reordered with respect to its parent and then how it is reordered with respect to its siblings.

Based on these previous approaches, we propose to characterize contextual syntactic roles of a word in terms of POS tags of the words themselves and their relatives in a dependency tree. It is straightforward to incorporate parent information since each node has a unique parent. As for siblings information, we incorporate POS tags of the closest sibling to the left and the closest to the right. We do not include all of the siblings to avoid overfitting. In addition to these basic syntactic relations, we consider the grandparent relation.

The following list is a summary of the source contextual functions that we use. We describe a function with respect to the kind of contextual property of a word it returns: (i) the word itself (Lex); (ii) POS label of the word (Pos); (iii) POS label of the word's parent; (iv) POS of the word's closest sibling to the left, concatenated with the POS tag of the closest sibling to the right; (v) the POS label of the word's grandparent. We use target-side contextual functions returning: (i) an empty string, (ii) POS of the word, (iii) the word itself.

Notation. We do not use the above functions separately to define individual BiLM models, but use combinations of these functions. We use the following notation for function combinations: "•" horizontally connects source (on the left) and target (on the right) contextual functions for a given model. For example, Lex $\bullet$ Lex refers to the original (lexicalized) BiLM. We use arrows $(\rightarrow)$ to designate parental information (the arrow goes from parent to child). Pos $\rightarrow$ Pos refers to a combination of a function returning the POS of a word and the POS of its parent (as in Figure 3). Pos $\rightarrow$ Pos $\rightarrow$ Pos is a combination of the previous with the function returning the grandparent's POS. Finally, we use + sibl to indicate the use of the sibling function described above: For example, Pos $\rightarrow$ Pos + sibl is a source function that returns the word's POS, its parent's POS and the POS labels of the closest siblings to left and right. ${ }^{6}$ Pos + sibl $\rightarrow$ Pos is a source function returning the word's own POS, the POS of a word's parent, and the POS tags of the parent's siblings (left- and right-adjacent).

Figure 4 represents the sentence from Figure 2 during decoding in a system with an integrated Pos $\rightarrow$ Pos $\rightarrow$ Pos + sibløLex feature. It shows the sequence of produced bilingual tokens and corresponding labels in the introduced notation.

\subsection{Training}

Training of dependency-based BiLMs consists of a sequence of extraction steps: After having produced word-alignments for a bitext (Section 4),

\footnotetext{
${ }^{6}$ In case there is no sibling on one of the sides, $\epsilon$ (empty word) is returned.
} 


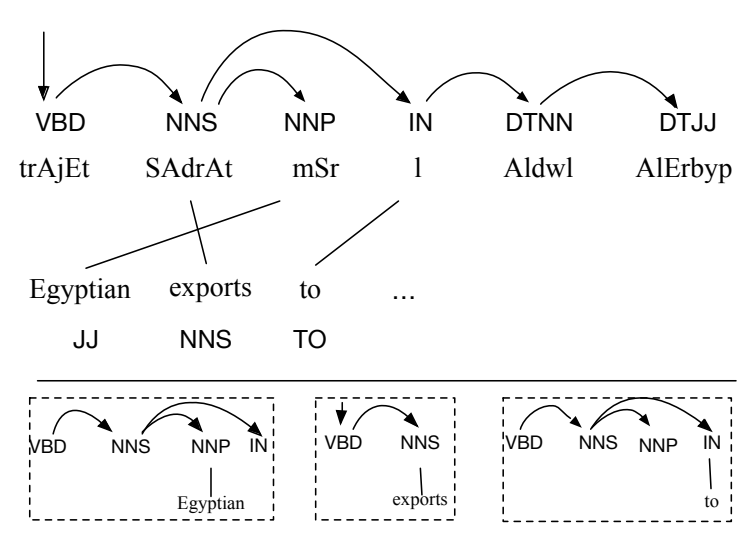

Figure 4: Sequence of bilingual tokens produced by a Pos $\rightarrow$ Pos $\rightarrow$ Pos + sibløLex after translating three words of the source sentence: $\mathrm{VBD} \rightarrow \mathrm{NNS} \rightarrow \epsilon+\mathrm{NNS}+\mathrm{IN} \bullet$ Egyptian, $\quad \mathrm{ROOT} \rightarrow \mathrm{VBD} \rightarrow$ $\epsilon+\mathrm{NNS}+\epsilon \bullet$ exports, $\mathrm{VBD} \rightarrow \mathrm{NNS} \rightarrow \mathrm{NNP}+\mathrm{IN}+\epsilon \bullet$ to (if there is no sibling on either of the sides, $\epsilon$ is returned).

sentences are segmented according to Equation 3. We produce a dependency parse of a source sentence and a POS-tag labeling of a target sentence. For Chinese, we use the Stanford dependency parser (Chang et al., 2009). For Arabic a dependency parser is not available for public use, so we produce a constituency parse with the Stanford parser (Green and Manning, 2010) and extract dependencies based on the rules in Collins (1999). For English POS-tagging, we use the Stanford POS-tagger (Toutanova et al., 2003). After having produced a labeled sequence of tokens, we learn a 5-gram model using SRILM (Stolcke et al., 2011). Kneyser-Ney smoothing is used for all model variations except for Pos•Pos where Witten-Bell smoothing is used due to zero countof-counts.

\subsection{Decoder integration}

Dependency-based BiLMs are integrated into our phrase-based SMT decoder as follows: Before translating a sentence, we produce its dependency parse. Phrase-internal word-alignments, needed to segment the translation hypothesis into tokens, are stored in the phrase table, based on the most frequent internal alignment observed during training. Likewise, we store the most likely target-side POS-labeling for each phrase pair.

The decoding algorithm is augmented with one additional feature function and one additional, corresponding feature weight. At each step of the derivation, as a new phrase pair is added to the

\begin{tabular}{|l|l|r|}
\hline Training set & N. of lines & N. of tokens \\
\hline Source side of Ar-En set & $4,376,320$ & $148 \mathrm{M}$ \\
Target side of Ar-En set & $4,376,320$ & $146 \mathrm{M}$ \\
\hline Source side of Ch-En set & $2,104,652$ & $20 \mathrm{M}$ \\
Target side of Ch-En set & $2,104,652$ & $28 \mathrm{M}$ \\
\hline
\end{tabular}

Table 1: Training data for Arabic-English and Chinese-English experiments.

partial translation hypothesis, this function segments the new phrase into bilingual tokens (given the internal alignment information) and substitutes the words in the phrase pair with syntactic labels (given the source parse and the target POS labeling associated with the phrase). The new syntactified bilingual tokens are added to the stack of preceding $n-1$ tokens, and the feature function computes the weighted updated model probability. During decoding, the probabilities of the BiLMs are computed in a stream-based fashion, with bilingual tokens as string tokens, and not in a class-based fashion, with syntactic source-side representations emitting the corresponding target words (Bisazza and Monz, 2014).

\section{Experiments}

\subsection{Setup}

We conduct translation experiments with a baseline PBSMT system with additionally one of the dependency-based BiLM feature functions specified in Section 3. We compare the translation performance to a baseline PBSMT system and to a baseline augmented with the original BiLMs from (Niehues et al., 2011).

Word-alignment is produced with GIZA++ (Och and Ney, 2003). We use an in-house implementation of a PBSMT system similar to Moses (Koehn et al., 2007). Our baseline contains all standard PBSMT features including language model, lexical weighting, and lexicalized reordering. The distortion limit is set to 5. A 5-gram LM is trained on the English Gigaword corpus (1.6B tokens) using SRILM with modified Kneyser-Ney smoothing and interpolation. The BiLMs were trained as described in Section 3.3. Information about the parallel data used for training the Arabic-English $^{7}$ and Chinese-English systems ${ }^{8}$ is

${ }^{7}$ The following Arabic-English parallel corpora were used: LDC2006E25, LDC2004T18, several gale corpora, LDC2004T17, LDC2005E46, LDC2007T08, LDC2004E13.

${ }^{8}$ The following Chinese-English parallel corpora were used: LDC2002E18, LDC2002L27, LDC2003E07, LDC2003E14, LDC2005T06, LDC2005T10, LDC2005T34, 


\begin{tabular}{|c|c|c|c|c|c|c|c|}
\hline & \multirow[t]{2}{*}{ Configuration } & \multicolumn{2}{|c|}{ MT08 } & \multicolumn{2}{|c|}{ MT09 } & \multicolumn{2}{|c|}{ MT08+MT09 } \\
\hline & & BLEU & TER & BLEU & TER & BLEU & TER \\
\hline $\mathrm{a}$ & PBSMT baseline & 45.12 & 47.94 & 48.16 & 44.30 & 46.57 & 46.21 \\
\hline \multirow[t]{2}{*}{$\mathrm{b}$} & Lex $\bullet$ Lex & 45.27 & 47.79 & $48.85^{\mathbf{\Lambda}}$ & $43.96^{\triangle}$ & $46.98^{\wedge}$ & $45.96^{\triangle}$ \\
\hline & Pos $\bullet$ Pos & 44.80 & 47.84 & 48.22 & $44.14^{\Delta,-}$ & 46.44 & 46.07 \\
\hline $\mathrm{c}$ & Pos $\rightarrow$ Pos $\bullet$ Pos & $45.66^{\mathbf{\Lambda}, \Delta}$ & $47.17^{\Lambda, \Lambda}$ & $49.00^{\mathbf{\Lambda},-}$ & $43.45^{\Lambda, \Lambda}$ & $47.25^{\mathbf{\Lambda}, \Delta}$ & $45.40^{\Lambda, \Lambda}$ \\
\hline $\mathrm{d}$ & Pos $\rightarrow$ Pos - sibl $\bullet$ Pos & $45.46^{\Delta,-}$ & $47.45^{\mathbf{\Lambda}, \Delta}$ & $48.69^{\boldsymbol{\Lambda},-}$ & $43.64^{\boldsymbol{\Lambda}, \Delta}$ & $47.00^{\mathbf{\Lambda},-}$ & $45.64^{\mathbf{\Lambda},-}$ \\
\hline $\mathrm{e}$ & Pos $\rightarrow$ Pos $\rightarrow$ Pos $\bullet$ Pos & $45.68^{\Lambda, \Delta}$ & $47.42^{\mathbf{\Lambda}, \Delta}$ & $49.09^{\mathbf{\Lambda},-}$ & $43.59^{\mathbf{\Lambda}, \boldsymbol{\Lambda}}$ & 47.30 $1, \Delta$ & $45.60^{\mathbf{\Lambda}, \boldsymbol{\Lambda}}$ \\
\hline$\overline{\mathrm{f}}$ & Lex $\bullet$ Lex + Pos $\rightarrow$ Pos $\rightarrow$ Pos $\bullet$ Pos & $45.63^{\mathbf{\Lambda}, \Delta}$ & $47.48^{\mathbf{\Lambda}, \Delta}$ & $49.30^{\Lambda, \Lambda}$ & $43.60^{\mathbf{\Lambda}, \Delta}$ & $47.38^{\Lambda, \Lambda}$ & $45.63^{\boldsymbol{\Lambda}, \boldsymbol{\Lambda}}$ \\
\hline
\end{tabular}

Table 2: BLEU and TER scores for Arabic-English experiments. Statistically significant improvements over the baseline (a) are marked $\boldsymbol{\Delta}$ at the $p<.01$ level and ${ }^{\Delta}$ at the $p<.05$ level. Additionally, ${ }^{\circ} \boldsymbol{\Delta}$ and $\cdot, \triangle$ indicate significant improvements with respect to BiLM Lex $\bullet$ Lex (b). Since TER is an error rate, lower scores are better.

\begin{tabular}{|c|c|c|c|c|c|c|}
\hline \multirow[t]{2}{*}{ Configuration } & \multicolumn{2}{|c|}{ MT08 } & \multicolumn{2}{|c|}{ MT09 } & \multicolumn{2}{|c|}{ MT08+MT09 } \\
\hline & BLEU & TER & BLEU & TER & BLEU & TER \\
\hline Pos $\rightarrow$ Pos $\bullet \epsilon$ & $45.66^{\boldsymbol{\Lambda}, \Delta}$ & $47.44^{\mathbf{\Lambda}, \Delta}$ & $48.78^{\mathbf{\Lambda}}$ & $43.94^{\mathbf{\Lambda},-}$ & $47.15^{\mathbf{\Lambda}}$ & $45.77^{\boldsymbol{\Lambda}, \Delta}$ \\
\hline Pos $\rightarrow$ Pos $\bullet$ Pos & $45.66^{\mathbf{\Lambda}, \Delta}$ & $47.17^{\Lambda, \Lambda}$ & $49.00^{\mathbf{\Lambda}}$ & $43.45^{\perp,}$ & $47.25^{\mathbf{\Lambda}, \Delta}$ & $45.40^{\Lambda, \Lambda}$ \\
\hline Pos $\rightarrow$ Pos $\bullet$ Lex & $45.48^{\triangle,-}$ & $47.34^{\mathbf{\Lambda}, \boldsymbol{\Lambda}}$ & $48.90^{\mathbf{\Lambda}}$ & $43.87^{\mathbf{\Lambda}, \Delta}$ & $47.12^{\mathbf{\Lambda}}$ & $45.69^{\mathbf{x}}$ \\
\hline
\end{tabular}

Table 3: Different combinations of a target contextual function with the Pos $\rightarrow$ Pos source contextual function for Arabic-English. See Table 2 for the notation regarding statistical significance.

shown in Table 1.

The feature weights were tuned by using pairwise ranking optimization (Hopkins and May, 2011) on the MT04 benchmark (for both language pairs). During tuning, 14 PRO parameter estimation runs are performed in parallel on different samples of the n-best list after each decoder iteration. The weights of the individual PRO runs are then averaged and passed on to the next decoding iteration. Performing weight estimation independently for a number of samples corrects for some of the instability that can be caused by individual samples. For testing, we used MT08 and MT09 for Arabic, and MT06 and MT08 for Chinese. We use approximate randomization (Noreen, 1989; Riezler and Maxwell, 2005) to test for statistically significant differences.

In the next two subsections we discuss the general results for Arabic and Chinese, where we use case-insensitive BLEU (Papineni et al., 2002) and TER (Snover et al., 2006) as evaluation metrics. This is followed by a preliminary analysis of observed reorderings where we compare 4-gram precision results and conduct experiments with an increased distortion limit.

\subsection{Arabic-English translation experiments}

We are interested in how a translation system with an integrated dependency-based BiLM fea-

and several gale corpora. ture performs as compared to the standard PBSMT baseline and, more importantly, to the original BiLM model. We consider two variants of BiLM discussed by Niehues et al. (2011): the standard one, Lex $\bullet$ Lex, and the simplest syntactic one, Pos•Pos. Results for the experiments can be found in Table 2. In the discussion below we mostly focus on the experimental results for the large, combined test set MT08+MT09.

Table 2.a-b compares the performance of the baseline and original BiLM systems. Lex•Lex yields strongly significant improvements over the baseline for BLEU and weakly significant improvements for TER. Therefore, for the rest of the experiments we are interested in obtaining further improvements over Lex॰Lex.

Pos $\rightarrow$ Pos•Pos (Table 2.c) demonstrates the effect of adding minimal dependency information to a BiLM. ${ }^{9}$ It results in strongly significant improvements over the baseline and weak improvements over Lex $\bullet$ Lex in terms of BLEU. We additionally ran experiments with the different target functions (Table 3). •Pos shows the highest results, and $\bullet \epsilon$ the lowest ones: this implies that a rather expressive source syntactic representation alone still benefits from target-side syntactic information. Below, our dependency-based systems only use $\bullet$ Pos.

Next, we tested the effect of adding more source

\footnotetext{
${ }^{9}$ Additional significance testing, which is not shown in Table 2, shows a strongly significant improvement over the original syntactic BiLM Pos•Pos.
} 


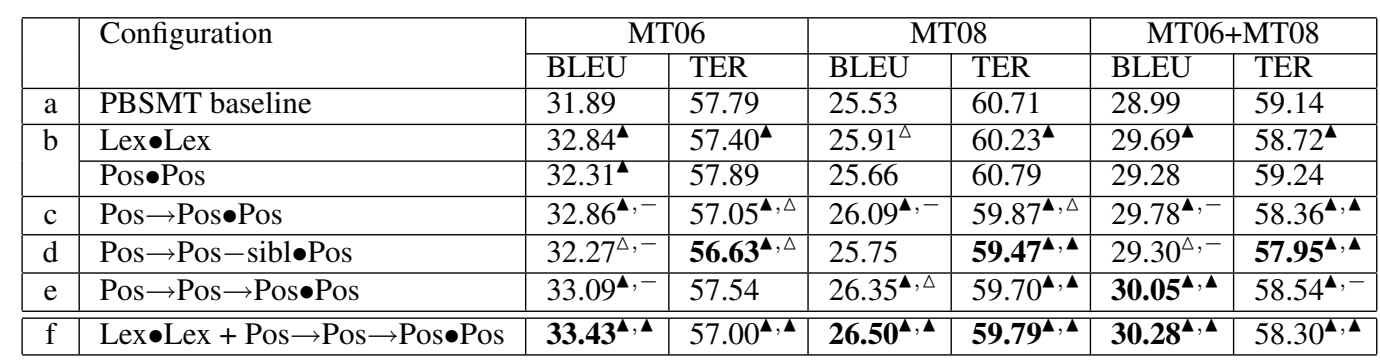

Table 4: BLEU and TER scores for Chinese-English PBSMT baseline and BiLM pipelines. See Table 2 for the notation regarding statistical significance.

\begin{tabular}{|c|c|c|c|c|c|c|}
\hline \multirow[t]{2}{*}{ Configuration } & \multicolumn{2}{|c|}{ MT06 } & \multicolumn{2}{|c|}{ MT08 } & \multicolumn{2}{|c|}{ MT06+MT08 } \\
\hline & BLEU & TER & BLEU & TER & BLEU & TER \\
\hline $\mathrm{Pos} \rightarrow \operatorname{Pos} \bullet \epsilon$ & $32.43^{\mathbf{\Lambda}}$ & $57.42^{\mathbf{\Lambda}}$ & 25.84 & 60.51 & $29.43^{\mathbf{\Lambda}}$ & $58.86^{\boldsymbol{\Lambda}}$ \\
\hline Pos $\rightarrow$ Pos $\bullet$ Pos & $32.86^{\mathbf{A},-}$ & $57.05^{\mathbf{\Lambda}, \triangle}$ & $26.09^{\mathbf{4},-}$ & $59.87^{\boldsymbol{\Lambda}, \triangle}$ & $29.78^{\mathbf{A}}$ & $58.36^{\boldsymbol{\Lambda}, \boldsymbol{\Lambda}}$ \\
\hline Pos $\rightarrow$ Pos $\bullet$ Lex & $32.69^{\mathbf{x}}$ & $57.03^{\mathbf{\Delta}, \triangle}$ & 25.72 & $60.17^{\mathbf{\Lambda},-}$ & $29.52^{\boldsymbol{A}}$ & $58.49^{\mathbf{\Lambda}, \triangle}$ \\
\hline
\end{tabular}

Table 5: Different combinations of a target contextual function with the Pos $\rightarrow$ Pos source contextual function for Chinese-English. See Table 2 for the notation regarding statistical significance.

dependency information. Pos $\rightarrow$ Pos + sibl•Pos (Table 2.d) only improves over the PBSMT baseline (but also shows weak improvements over Lex•Lex for TER). It significantly degrades the performance with respect to the Pos $\rightarrow$ Pos $\bullet$ Pos system (Table 2.c). Pos $\rightarrow$ Pos $\rightarrow$ Pos $\bullet$ Pos (Table 2.e) shows the best results overall for BLEU, although it must be pointed out that the difference with Pos $\rightarrow$ Pos $\bullet P o s$ is very small. With respect to TER, Pos $\rightarrow$ Pos $\bullet P o s$ outperforms the grandparent variant.

So far, we can conclude that source parent information helps improve translation performance. Increased specificity of a parent (parent specified by a grandparent) tends to further improve performance. Up to now, we have only used syntactic information and obtained considerable improvements over Pos•Pos, surpassing the improvement provided by Lex $\bullet$ Lex. Can we gain further improvements by also adding lexical information? To this end, we conduct experiments combining the best performing dependency-based BiLM (Pos $\rightarrow$ Pos $\rightarrow$ Pos $\bullet P o s)$ and the lexicalized BiLM (Lex॰Lex). We hypothesize that the two models improve different aspects of translation: Lex $\bullet$ Lex is biased towards improving lexical choice and Pos $\rightarrow$ Pos $\rightarrow$ Pos $\bullet$ Pos towards improving reordering. Combining these two models, we may improve both aspects. The metric results for the combined set indeed support this hypothesis (Table 2.f).

\subsection{Chinese-English translation experiments}

The results of the Chinese-English experiments are shown in Table 4. In the discussion below we mostly focus on the experimental results for the large, combined test set MT06+MT08. We observe the same general pattern for the Pos $\rightarrow$ Pos source function (Table 4.c) as for Arabic-English: the system with the $\bullet$ Pos target function has the highest scores (Table 5). All of the Pos $\rightarrow$ Pos• configurations show statistically significant improvements over the PBSMT baseline. For TER, two of the three Pos $\rightarrow$ Pose variants significantly outperform Lex $\bullet$ Lex. The system with sibling information (Table 4.d) obtains quite low BLEU results, just as in the Arabic experiments. On the other hand, its TER results are the highest overall. The system with the Pos $\rightarrow$ Pos $\rightarrow$ Pos $\bullet$ Pos function (Table 4.e) achieves the best results among dependency-based BiLMs for BLEU. Finally, combining Pos $\rightarrow$ Pos $\rightarrow$ Pos $\bullet$ Pos and Lex $\bullet$ Lex results in the largest and significant improvements over all competing systems for BLEU.

\subsection{Preliminary analysis of reordering in translation experiments}

In general, the experimental results show that using source dependency information yields consistent improvements for translating from Arabic and Chinese into English. On the other hand, we have pointed out some discrepancies between the two metrics employed, suggesting that different system configurations may improve different aspects 


\begin{tabular}{|c|c|c|c|c|c|c|c|}
\hline & Configuration & \multicolumn{3}{|c|}{ Ar-En } & \multicolumn{3}{|c|}{ Ch-En } \\
\hline & & MT08 & MT09 & MT08+MT09 & MT06 & MT08 & MT06+MT08 \\
\hline $\mathrm{a}$ & PBSMT baseline & 26.14 & 29.81 & 27.88 & 14.48 & 10.96 & 12.89 \\
\hline $\mathrm{b}$ & Lex•Lex & 26.33 & 30.55 & 28.32 & 15.43 & 11.45 & 13.65 \\
\hline & Pos $\bullet$ Pos & 25.95 & 30.06 & 27.89 & 14.76 & 11.01 & 13.07 \\
\hline $\mathrm{c}$ & Pos $\rightarrow$ Pos $\bullet$ Pos & 26.91 & 31.08 & 28.87 & 15.29 & 11.52 & 13.60 \\
\hline $\mathrm{e}$ & Pos $\rightarrow$ Pos - sibl•Pos & 26.71 & 30.73 & 28.60 & 15.27 & 11.67 & 13.66 \\
\hline $\mathrm{d}$ & $\mathrm{Pos} \rightarrow \mathrm{Pos} \rightarrow \mathrm{Pos} \bullet \mathrm{Pos}$ & 26.78 & 31.09 & 28.80 & 15.42 & 11.70 & 13.77 \\
\hline$\overline{f f}$ & Lex $\bullet$ Lex + Pos $\rightarrow$ Pos $\rightarrow$ Pos $\bullet$ Pos & 26.80 & 31.27 & 28.90 & $\overline{15.87}$ & $\overline{11.85}$ & $\overline{14.07}$ \\
\hline
\end{tabular}

Table 6: 4-gram precision scores for Arabic-English and Chinese-English baseline and BiLM systems.

\begin{tabular}{|c|c|c|c|c|c|c|c|c|c|}
\hline \multirow[t]{2}{*}{ Configuration } & \multicolumn{3}{|c|}{ MT08 } & \multicolumn{3}{|c|}{ MT09 } & \multicolumn{3}{|c|}{ MT08+MT09 } \\
\hline & BLEU & TER & 4gram & BLEU & TER & 4gram & BLEU & TER & 4gram \\
\hline Lex $\bullet$ Lex & 45.19 & 47.06 & 26.41 & 48.39 & 44.11 & 30.23 & 46.72 & 45.97 & 28.21 \\
\hline $\mathrm{Pos} \rightarrow \mathrm{Pos} \rightarrow \mathrm{Pos} \bullet \mathrm{Pos}$ & 45.49 & $47.31^{\triangle}$ & 26.66 & $48.90^{\mathbf{4}}$ & $43.57^{\mathbf{A}}$ & 30.92 & $47.12^{\mathbf{A}}$ & $45.52^{\mathbf{x}}$ & 28.66 \\
\hline
\end{tabular}

Table 7: BLEU, TER and 4-gram precision scores for Arabic-English Lex $\bullet$ Lex and Pos $\rightarrow \mathrm{Pos} \rightarrow \mathrm{Pos} \bullet \mathrm{Pos}$ with a distortion limit of 10 .

\begin{tabular}{|c|c|c|c|c|c|c|c|c|c|}
\hline \multirow[t]{2}{*}{ Configuration } & \multicolumn{3}{|c|}{ MT06 } & \multicolumn{3}{|c|}{ MT08 } & \multicolumn{3}{|c|}{ MT06+MT08 } \\
\hline & $\overline{B L E U}$ & TER & 4gram & BLEU & TER & 4gram & BLEU & TER & 4gram \\
\hline Lex $\bullet$ Lex & 33.26 & 56.81 & 16.06 & 25.67 & 60.19 & 11.42 & 29.79 & 58.38 & 13.96 \\
\hline $\mathrm{Pos} \rightarrow \mathrm{Pos} \rightarrow \mathrm{Pos} \bullet \mathrm{Pos}$ & $33.92^{\star}$ & $56.29^{4}$ & 16.26 & $27.00^{4}$ & $59.58^{\star}$ & 12.26 & $30.77^{\mathbf{\Lambda}}$ & $57.82^{\star}$ & 14.46 \\
\hline
\end{tabular}

Table 8: BLEU, TER and 4-gram precision scores for Chinese-English Lex॰Lex and $\mathrm{Pos} \rightarrow \mathrm{Pos} \rightarrow$ Pos $\bullet$ Pos with a distortion limit of 10 .

of translation. To this end, we conducted some additional evaluations to understand how reordering is affected by the proposed features.

We use 4-gram precision as a metric of how much of the reference set word order is preserved. Table 6 shows the corresponding results for both languages. Just as in the previous two sections, configurations with parental information produce the best results. For Arabic, all of the dependency configurations outperform Lex $\bullet$ Lex. But the system with two feature functions, one of which is Lex॰Lex, still obtains the best results, which may suggest that the lexicalized BiLM also helps to differentiate between word orders. For Chinese, Pos $\rightarrow$ Pos $\rightarrow$ Pos $\bullet$ Pos and the system combining the latter and Lex $\bullet$ Lex also obtain the best results. However, other dependency-based configurations do not outperform Lex $\bullet$ Lex.

All the experiments so far were run with a distortion limit of 5. But both of the languages, especially Chinese, often require reorderings over a longer distance. We performed additional experiments with a distortion limit of 10 for the Lex•Lex and Pos $\rightarrow$ Pos $\rightarrow$ Pos $\bullet$ Pos systems (Tables 7 and 8). It is more difficult to translate with a higher distortion limit (Green et al., 2010) as the set of permutations grows larger thereby making it more difficult to differentiate between correct and incorrect continuations of the current hypothesis. It has also been noted that higher distortion limits are more likely to result in improvements for Chinese rather than Arabic to English translation (Chiang, 2007; Green et al., 2010).

We compared performance of fixed BiLM models at distortion lengths of 5 and 10. ArabicEnglish results did not reveal statistically significant differences between the two distortion limits for Pos $\rightarrow$ Pos $\rightarrow$ PosePos. On the other hand, for Lex Lex BLEU decreases when using a distortion limit of 10 compared to a limit of 5 . This implies that the dependency BiLM is more robust in the more challenging reordering setting than the lexicalized BiLM. Chinese-English results for Pos $\rightarrow$ Pos $\rightarrow$ Pos $\bullet$ Pos do show significant improvements over the distortion limit of 5 (up to 0.49 BLEU higher than the best result in Table 4). This indicates that the dependency-based BiLM is better capable to take advantage of the increased distortion limit and discriminate between correct and incorrect reordering choices.

Comparing the results for Pos $\rightarrow \mathrm{Pos} \rightarrow \mathrm{Pos} \bullet P o s$ and Lex Lex at a distortion limit of 10 , we obtain strongly significant improvements for all metrics. For Chinese, a larger distortion limit helps for both configurations, but more so for our dependency BiLM, yielding an improvement of 0.98 BLEU 
over the original, lexicalized BiLM (Table 8).

\section{Conclusions}

In this paper, we have introduced a simple, yet effective way to include syntactic information into phrase-based SMT. Our method consists of enriching the representation of units of a bilingual language model (BiLM). We argued that the very limited contextual information used in the original bilingual models (Niehues et al., 2011) can capture reorderings only to a limited degree and proposed a method to incorporate information from a source dependency tree in bilingual units. In a series of translation experiments we performed a thorough comparison between various syntacticallyenriched BiLMs and competing models. The results demonstrated that adding syntactic information from a source dependency tree to the representations of bilingual tokens in an n-gram model can yield statistically significant improvements over the competing systems.

A number of additional evaluations provided an indication for better modeling of reordering phenomena. The proposed dependency-based BiLMs resulted in an increase in 4-gram precision and provided further significant improvements over all considered metrics in experiments with an increased distortion limit.

In this paper, we have focused on rather elementary dependency relations, which we are planning to expand on in future work. Our current approach is still strictly tied to the number of target tokens. In particular, we are interested in exploring ways to better capture the notion of syntactic cohesion in translation (Fox, 2002; Cherry, 2008) within our framework.

\section{Acknowledgments}

We thank Arianna Bisazza and the reviewers for their useful comments. This research was funded in part by the Netherlands Organization for Scientific Research (NWO) under project numbers 639.022.213 and 612.001.218.

\section{References}

Yaser Al-Onaizan and Kishore Papineni. 2006. Distortion models for statistical machine translation. In Proceedings of the 21st International Conference on Computational Linguistics and 44th Annual Meeting of the Association for Computational Linguis- tics, pages 529-536, Sydney, Australia, July. Association for Computational Linguistics.

Alexandra Birch. 2011. Reordering Metrics for Statistical Machine Translation. Ph.D. thesis, University of Edinburgh.

Arianna Bisazza and Christof Monz. 2014. Classbased language modeling for translating into morphologically rich languages. In Proceedings of the 25th International Conference on Computational Linguistics (COLING 2014), pages 19181927, Dublin, Ireland, August.

Pi-Chuan Chang, Huihsin Tseng, Dan Jurafsky, and Christopher D. Manning. 2009. Discriminative reordering with chinese grammatical relations features. In Proceedings of the Third Workshop on Syntax and Structure in Statistical Translation, pages 51-59. Association for Computational Linguistics.

Colin Cherry. 2008. Cohesive phrase-based decoding for statistical machine translation. In Proceedings of Association for Computational Linguistics, pages 72-80.

David Chiang. 2007. Hierarchical phrase-based translation. Computational Linguistics, 33(2):201-228.

David Chiang. 2010. Learning to translate with source and target syntax. In Proceedings of the 48th Annual Meeting of the Association for Computational Linguistics, pages 1443-1452. Association for Computational Linguistics.

Michael Collins. 1999. Head-Driven Statistical Models for Natural Language Parsing. Ph.D. thesis, University of Pennsylvania.

Josep M. Crego and François Yvon. 2010a. Factored bilingual n-gram language models for statistical machine translation. Machine Translation, 24(2):159175.

Josep M. Crego and François Yvon. 2010b. Improving reordering with linguistically informed bilingual n-grams. In Proceedings of the 23rd International Conference on Computational Linguistics, pages 197-205. Association for Computational Linguistics.

Nadir Durrani, Helmut Schmid, and Alexander Fraser. 2011. A joint sequence translation model with integrated reordering. In Proceedings of the 49th Annual Meeting of the Association for Computational Linguistics, pages 1045-1054. Association for Computational Linguistics.

Heidi J. Fox. 2002. Phrasal cohesion and statistical machine translation. In Proceedings of the ACL02 conference on Empirical methods in natural language processing, pages 304-3111. Association for Computational Linguistics. 
Michel Galley and Christopher D. Manning. 2008. A simple and effective hierarchical phrase reordering model. In Proceedings of the Conference on Empirical Methods in Natural Language Processing, pages 848-856. Association for Computational Linguistics.

Spence Green and Christopher D. Manning. 2010. Better arabic parsing: Baselines, evaluations, and analysis. In Proceedings of the 23rd International Conference on Computational Linguistics, pages 394-402. Association for Computational Linguistics.

Spence Green, Michel Galley, and Christopher D. Manning. 2010. Improved models of distortion cost for statistical machine translation. In Proceedings of the 2010 Annual Conference of the North American Chapter of the Association for Computational Linguistics, pages 867-875. Association for Computational Linguistics.

Mark Hopkins and Jonathan May. 2011. Tuning as ranking. In Proceedings of the Conference on Empirical Methods in Natural Language Processing, pages 1352-1362. Association for Computational Linguistics.

Liang Huang and Haitao Mi. 2010. Efficient incremental decoding for tree-to-string translation. In Proceedings of the 2010 Conference on Empirical Methods in Natural Language Processing, pages 273-283. Association for Computational Linguistics.

Liang Huang, Kevin Knight, and Aravind Joshi. 2006. Statistical syntax-directed translation with extended domain of locality. In Proceedings of AMTA, pages 223-226.

Philipp Koehn, Franz Josef Och, and Daniel Marcu. 2003. Statistical phrase-based translation. In Proceedings of the 2003 Conference of the North American Chapter of the Association for Computational Linguistics on Human Language Technology, pages 48-54. Association for Computational Linguistics.

Philipp Koehn, Hieu Hoang, Alexandra Birch, Chris Callison-Burch, Marcello Federico, Nicola Bertoldi, Brooke Cowan, Wade Shen, Christine Moran, Richard Zens, et al. 2007. Moses: Open source toolkit for statistical machine translation. In Proceedings of the 45th Annual Meeting of the Association for Computational Linguistics on Interactive Poster and Demonstration Sessions, pages 177-180. Association for Computational Linguistics.

Uri Lerner and Slav Petrov. 2013. Source-side classifier preordering for machine translation. In Proceedings of the Empirical Methods in Natural Language Processing.

Junhui Li, Zhaopeng Tu, Guodong Zhou, and Josef van Genabith. 2012. Head-driven hierarchical phrasebased translation. In Proceedings of the 50th Annual Meeting of the Association for Computational
Linguistics, pages 33-37. Association for Computational Linguistics.

Yang Liu, Qun Liu, and Shouxun Lin. 2006. Treeto-string alignment template for statistical machine translation. In Proceedings of the 21st International Conference on Computational Linguistics and the 44th annual meeting of the Association for Computational Linguistics, pages 609-616. Association for Computational Linguistics.

José B Marino, Rafael E Banchs, Josep M. Crego, Adria de Gispert, Patrik Lambert, José A.R. Fonollosa, and Marta R. Costa-Jussà. 2006. N-grambased machine translation. Computational Linguistics, 32(4):527-549.

Yuval Marton and Philip Resnik. 2008. Soft syntactic constraints for hierarchical phrased-based translation. In Proceedings of the Association for Computational Linguistics, pages 1003-1011.

Jan Niehues, Teresa Herrmann, Stephan Vogel, and Alex Waibel. 2011. Wider context by using bilingual language models in machine translation. In Proceedings of the Sixth Workshop on Statistical Machine Translation, pages 198-206. Association for Computational Linguistics.

Eric W. Noreen. 1989. Computer Intensive Methods for Testing Hypotheses. An Introduction. WileyInterscience.

Franz Josef Och and Hermann Ney. 2003. A systematic comparison of various statistical alignment models. Computational Linguistics, 29(1):19-51.

Kishore Papineni, Salim Roukos, Todd Ward, and WeiJing Zhu. 2002. Bleu: a method for automatic evaluation of machine translation. In Proceedings of the 40th annual meeting of the Association for Computational Linguistics, pages 311-318. Association for Computational Linguistics.

Stefan Riezler and John T. Maxwell. 2005. On some pitfalls in automatic evaluation and significance testing for MT. In Proceedings of the ACL Workshop on Intrinsic and Extrinsic Evaluation Measures for Machine Translation and/or Summarization.

Libin Shen, Jinxi Xu, and Ralph M. Weischedel. 2008. A new string-to-dependency machine translation algorithm with a target dependency language model. In Proceedings of the Association for Computational Linguistics, pages 577-585.

Matthew Snover, Bonnie Dorr, Richard Schwartz, Linnea Micciulla, and John Makhoul. 2006. A study of translation edit rate with targeted human annotation. In Proceedings of AMTA, pages 223-231.

Andreas Stolcke, Jing Zheng, Wen Wang, and Victor Abrash. 2011. Srilm at sixteen: Update and outlook. In Proceedings of IEEE Automatic Speech Recognition and Understanding Workshop, page 5. 
Christoph Tillmann. 2004. A unigram orientation model for statistical machine translation. In Proceedings of of the North American Chapter of the Association for Computational Linguistics, pages 101-104. Association for Computational Linguistics.

Kristina Toutanova, Dan Klein, Christopher D. Manning, and Yoram Singer. 2003. Feature-rich part-ofspeech tagging with a cyclic dependency network. In Proceedings of the 2003 Conference of the North American Chapter of the Association for Computational Linguistics on Human Language Technology, pages 173-180. Association for Computational Linguistics.

Richard Zens and Hermann Ney. 2003. A comparative study on reordering constraints in statistical machine translation. In Proceedings of the 41st Annual Meeting on Association for Computational LinguisticsVolume 1, pages 144-151. Association for Computational Linguistics. 